except the part behind the screen. It is essential that the switchboard be of the dual control type allowing a rapid change-over from the screening to the exposure position. The radiologist controls the screening current by means of a foot-switch while a radiographer is in readiness to make the exposure. During screening the casette is at the side of the screen and protected from the rays by the lead. At the appropriate moment the radiologist cuts off the screening current, calls " Ready," pushes the film behind the screen, and calls "Film" as a sign for the exposure to be made. During the short period between the signals "Ready" and "Film" the radiographer switches over from the screening to the exposure position. The advantages of this method are that the interval between screening and exposure is reduced to the minimum and that the radiologist can see the actual exposure on the screen and so knows whether it is correct or not. If necessary a compressor of loofah or other material can be attached; but I prefer to use the gloved hand, by means of which a finely graduated degree of pressure can be applied from below without obscuring the picture.

Apart from the screen the apparatus was made at a cost of a few shillings. Rarely are more than two or at the most three films necessary for the adequate demonstration of an ulcer. I suggest that if this simple device were adopted at all the Service hospitals the annual saving to the Exchequer would be considerable.

\section{THE CONTROL OF LICE}

\section{BY}

P. A. BUXTON, M.R.C.S., L.R.C.P.

Professor of Entomology, London School of Hygiene and Tropical Medicine, University of London

Now that a considerable number of our people are sleeping crowded together in shelters it is likely that outbreaks of body and head lice will occur. Every medical man should know something of the insect's biology and the methods of control. The points which appear most important are here summarized. A full account of the whole subject, including its epidemiological side, has been recently published (Buxton, 1939).

\section{Biology}

In all lice the adult female lays eggs (nits), from which larvae hatch: these resemble adults except that they are smaller. The larvae grow and become adult males or females. On human beings we speak of "head lice" and "body lice"; the points of difference are very slight, and the life history and control are essentially the same. . The "crab louse" or "pubic louse" is quite distinct from the others. All these three (head, body, and crab lice) are parasites of man and have no other host: every infestation comes from some other human being. The adults and larvae feed several times a day on blood alone. The whole life is generally passed close to the surface of the human body, the female sticking eggs to the hair of the head (head louse), or to the fibres of garments, particularly those next the skin, and to the hair of the trunk (body louse). Blankets are commonly infested with lice and eggs; furniture, walls, etc., so rarely that they are negligible.

At the temperature which prevails on the surface of the body (about $30^{\circ} \mathrm{C} ., 86^{\circ} \mathrm{F}$.) the egg stage lasts nine days, the larva lives nine days, and the adult about thirty days. The female lays eight to ten eggs a day-i.e., a total of about 250 eggs. A louse might, therefore, have 120 daughters and 14,400 granddaughters; even if the infant mortality is very high the rate of increase is surprisingly great after two or three generations.

Lice shun light, but probably move about rather freely at night. They spread readily when people are forced to live and sleep in a crowd, particularly if the washing of clothes and person is difficult. In England, in peacetime, I am convinced that more than $10 \%$ (and often over $30 \%$ ) of the children in elementary schools in towns and cities have head lice: in rural areas the figure is much lower. Girls are more often infested than boys of the same age; the infestation is higher in children of school age than in babies, adolescents, or adults. In peacetime the body louse is much rarer than the head louse, and is difficult to find except among the destitute. There is little doubt that it is more widely spread now than it was before the war, though no outbreaks have yet been recorded.

In any lousy community there is a tendency for most of the infested people to have no more than ten to twenty lice: a small proportion have a few hundred lice, and still fewer may have a few thousand. In other words, many infestations are so light that they may be missed, but a few of the people are centres of dissemination.

\section{Repellents and Preventives}

Essential oils and other volatile substances have a definite repellent effect, but not strong enough to prevent a louse sucking blood. We do not know any repellent which is of practical value. It is true that, given a choice, body lice will live and lay eggs on a rough material rather than a smooth (e:g., wool rather than silk), but this does not prevent them from colonizing silk underwear. It is definitely established that people with long hair are more liable to be infested with head lice than those with short: long hair is also more difficult to deal with if infested. Female social workers and others exposed to particular risk should sacrifice appearance and use the scissors freely.

\section{Control : General Points}

As head and body lice can multiply rapidly and spread through a crowded community, control is useless unless it is radical. If a group of men have body lice one must deal with their persons and clothes, and generally bedding. If it is possible the whole group-e.g., all the people who habitually sleep in one shelter-should be deloused in one day. Similarly with the head louse, one would wish to inspect and perhaps treat the infested child's neighbours in school, and his elder sister or infant brother.

More attention should be given to teaching people about the louse and ways of dealing with it, for some of the trouble is due to lack of frankness and to a tendency to conceal infestation. Make use of the poster published by the Central Council for Health Education, and the Health and Cleanliness Council's leaflet, "Cleanliness of School Children."

It is important to detect light infestations. If one is looking for head lice the eggs are generally easy to find: the living egg is plump and opalescent, and generally fixed to the hair close to the scalp; the dead egg or empty shell is flat and dirty, and may have been carried far from the scalp as the hair grew. Eggs are often fixed to hair in the region behind the ears; they may also be inside the hat. Most eggs of the body louse are fixed on seams or folds on the inner surface of undergarments; eggs and lice are generally congregated in limited areas. At the least one should examine shirt or vest, even if the subject is unwilling to strip completely. Some eggs and lice may also be found on the surface of the body, and on outer garments, even on their outer aspect.

\section{Control on the Person}

If the scalp is infested it is advisable, though not necessary, to shorten the hair. It is quite possible to disinfest a head bv thorough combing. giving particular 
attention to the base of the hair and using a metal comb with very fine teeth set so close that lice are combed out and eggs crushed: such a comb is sold by Messrs. Sacker. Success depends on thoroughness.

It is generally quicker to use an insecticidal liquid poured on (the patient lying on the back, with the head projecting over the edge of a table), or applied by dipping an ordinary comb in the material. The liquid must reach all parts of the hair, down to the scalp. Use one of the following:

(a) Paraffin (i.e., lamp oil, kerosene) 74 parts, cottonseed oil 20 parts, tar oil (wood tar) 5 parts, oil of lemon grass 1 part (the last being optional).

(b) Equal parts of kerosene and a vegetable oil (cottonseed, linseed, etc.). Some types of kerosene are more insecticidal than others, so that this mixture may be unsatisfactory.

(c) Carbolic acid (phenol) 1 in 40 of water.

(d) Cresols are also frequently used. I understand that liquor cresoli saponatus fortis ("army cresol") is more satisfactory than liquor cresoli saponatus (lysol), because army cresol contains a higher proportion of phenoloids, which are less irritating to the skin: "it is used $2 \%$, with $2 \%$ soft soap, in water. The composition of liquids (proprietary or other) which contain cresols is very complex, and perhaps variable: it might be well to test the available material for its effect both on the louse and on the human skin.

Whichever of these is used tie the head in a towel and leave it one hour: then wash. Which method is most effective is not known.

The two following are also efficacious: alcohol (rectified or methylated) 7, water 3 parts; extract of quassia chips, made with boiling water, and used fresh. With either of these there is no need to tie up the head, the liquid being allowed to dry off.

Insecticidal powders may well prove to be of great value. The one that was used extensively in the last war-N.C.I. $(96 \%$ naphthalene, $2 \%$ creosote, $2 \%$ iodoform) -is certainly effective against lice, but causes dermatitis on some people ; it therefore tends to be insufficiently applied, and then is condemned as ineffective. I have been allowed to watch the recent experiments of Messrs. H. J. Craufurd-Benson and J. .Macleod, entomologists to Messrs. Cooper, McDougall, and Robertson. They have tested a number of powders on infested tramps, whose garments were examined daily, and have produced two useful formulae, which will be published later. The first powder (known as AL. 16) is rather slow in taking effect, but otherwise very effective. The second (AL. 63) is similar but contains an additional constituent, which renders its action quicker, though it has a characteristic smell, which might be regarded as a disadvantage. Both powders hang about in garments, even if they are being worn, and continue to be effective for about a week. These powders appear equally suitable for the head or for garments, so far as can be judged at present. If used on the head the powder should be massaged down to the roots of the hair and excess brushed out, the eyes being shut. On garments, about $1 \mathrm{oz}$. suffices for shirt and pants, the powder being rubbed particularly into seams. As these powders are unlikely to kill nits two applications at a week's interval should be given.

Essential oils (including sassafras) are expensive and not particularly effective. The legend that vinegar or acetic acid loosens nits and enables them to be combed out was disproved twenty-five years ago: it dies hard. Many people think that dead nits are unsightly and must be removed. That is an aesthetic question-a matter for the hairdresser: to the doctor's eye a dead nit is a thing of beauty.

In dealing with the body louse, disinfest clothes, etc., as below: clip the hair, lather all over with soap and cresol (above), and take a bath. It is best to treat an infestation with crab lice in the same way: they generally occur on the pubic hair, but are not very rare in the scalp, axillae, etc.

Disinfestation of Garments, Blankets, etc.

The most convenient agent is heat. The following periods and temperatures are fatal to lice and eggs: five minutes $54^{\circ}$ C. $\left(129^{\circ}\right.$ F. $)$; forty-five minutes $50^{\circ}$ C. $\left(121^{\circ}\right.$ F. $)$.
It is immaterial whether the heat is dry or moist, but it is essential that the whole of the infested material should actually pass the necessary temperature. Cotton garments, if washed in the ordinary way, are exposed to temperatures which are lethal to lice and nits. Alternatively soak them in $2 \%$ cresol for at least an hour at $16^{\circ} \mathrm{C} .\left(61^{\circ} \mathrm{F}\right.$.) or higher temperature. Woollens shrink if washed in water hot enough to kill the louse, and are more conveniently treated by dry heat. In a disinfesting machine arrangements are made to allow for penetration of heat through blankets, coats, etc., which are hung on racks. If something has to be improvised the proper distribution of heat through the chamber and the disposal of the garments on racks need careful thought. Many types of heating have been used in improvised chambers-e.g., braziers, steam from an engine, etc. Garments can be disinfested with a domestic hot iron, attention being given to folds and pleats. One ironer can deal with the clothes of one person in the period occupied by the bath, etc. As a practical test, if eggs and collapsed and shrunk and if lice are brittle, all is well.

At present fumigation is not a method to recommend, the necessary doses and exposures not being well enough known.

As the louse cannot survive starvation for long, clothing may be disinfested by storage. At all temperatures the egg stage lasts longer than the starved adults or larvae, so that one must store long enough to allow all eggs to hatch, and a few days in addition to ensure that the newly hatched larvae die. The following facts are published by permission of my colleague $\mathrm{Mr}$. H. S. Leeson.

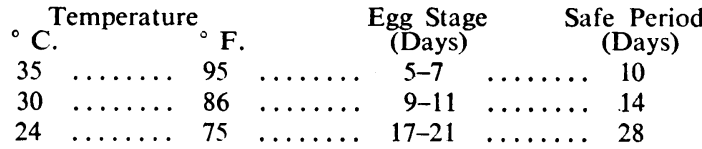

If maintained steadily at temperatures below $24^{\circ} \mathrm{C}$. the egg cannot hatch, though it can develop and hatch later if the temperature is raised. The safe period below $24^{\circ} \mathrm{C}$. is not exactly defined, but may be taken as a month.

\section{Organization}

The organization which is necessary, and the lay-out of a disinfesting station, are not easily dealt with in a brief paper. (See Army Manual of Hygiene or the book already quoted.)

As a minimum protection for sanitary personnel one suggests gum-boots ; two complete sets of clothes should be available, one being reserved for work and put. away in a closed box (e.g., metal dustbin) with a handful of flake naphthalene, the vapour of which kills lice. It might be necessary, particularly in the presence of an epidemic, to use a one-piece "louse-proof" garment of white washable material, reaching wrists and ankles and entered from the back.

\author{
REFERENCES \\ Army Manual of Hygiene, H.M.S.O., 1935 \\ Buxton, P. A. (1939). The Louse, Arnold, London.
}

M. Perrot (Thèse de Paris, 1940, No. 270), who records four cases in patients aged from 49 to 62 , states that the low incidence of cancer of the body of the uterus (4 to $5 \%$ of all uterine cancers), its restriction for months and even years to the uterine cavity, and the relative scarcity of lymphatics in the body of the uterus explain the rarity of metastases secondary to cancer in this site. Bone metastases of cancer of the body of the uterus are most frequent in the bones of the pelvis and hip-joint. There has often been too great a tendency to attribute these metastases to radiotherapy, but in some of these cases the metastases were already present before this treatment began. 